\title{
Reducing depressive symptoms and increasing positive feelings with expressive writing
}

\author{
Chen Sung Wong, Melissa Jane Chua, Kususanto Ditto Prihadi \\ Department of Psychology, HELP University, Kuala Lumpur, Malaysia
}

\begin{tabular}{|c|c|}
\hline Article Info & ABSTRACT \\
\hline Article history: & \multirow{8}{*}{$\begin{array}{l}\text { This experimental study examined the effect of expressive writing on } \\
\text { depressive symptoms. } 86 \text { undergraduate students were recruited from a } \\
\text { private university to perform online positive experience writing (PEW) } \\
\text { ( } \mathrm{n}=31 \text { ), negative experience (NEW) ( } \mathrm{n}=32 \text { ) and control topic }(\mathrm{CW})(\mathrm{n}=23) \\
\text { for three sessions in three consecutive weeks. The depressive symptoms of } \\
\text { participants were measured at pre-treatment and post-treatment. It was } \\
\text { hypothesized that PEW has significant greater reduction in depressive } \\
\text { symptoms compared to NEW and CW based on broaden and build model. } \\
\text { Nevertheless, results showed that PEW had significantly greater symptoms } \\
\text { reduction than CW, yet there was no significant difference between PEW and } \\
\text { NEW. The findings suggested that PEW might be potentially useful in } \\
\text { reducing depressive symptoms among undergraduates. }\end{array}$} \\
\hline Received Dec 18, 2020 & \\
\hline Revised Feb 24, 2021 & \\
\hline Accepted Mar 15, 2021 & \\
\hline Keywords: & \\
\hline Depressive symptoms & \\
\hline Expressive writing & \\
\hline Online intervention & \\
\hline
\end{tabular}

This is an open access article under the CC BY-SA license.

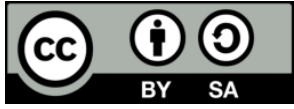

\section{Corresponding Author:}

Kususanto Ditto Prihadi

Department of Psychology

Higher Education Learning Philosophy (HELP) University

Selangor, Malaysia

Email:prihadi.k@help.edu.my

\section{INTRODUCTION}

In the past 30 years, numerous studies have been done on the effects of structured writing intervention on mental as well as physical health [1], [2]. The basic expressive writing paradigm involves the process of expressing thoughts and feelings about a personally stressful event or traumatic experience through disclosure writing [3]. In 1986, this writing paradigm was tested among 46 healthy university students, who were asked to write about their thoughts and feelings related to their personal traumatic experience for 20 minutes per session on four consecutive days. Students in expressive writing condition showed significantly fewer health center visits than students in control writing in a six months follow-up. The research has provided some preliminary insight on the range of physiological benefits of expressive writing.

In the broader context, studies in the last three decades showed that the practice of expressive writing can lead to improvement in lung and liver functioning [4], [5], decreased blood pressure [6] and even enhanced immune system functioning [7]. In addition, expressive writing also results in significant improvement in non-health related outcomes such as better academic performance [8], reduced absenteeism from work [4] and enhanced working memory [9]. From a psychological perspective, it was evident that expressive writing can lead to better psychological outcomes, such as improvement in post-traumatic growth [10], decreased tendency to ruminate [11] and diminished feelings of anger [12]. Some of the possible mechanisms involved might be emotional catharsis, emotional confrontation and repeated exposure through expressive writing [1]. However, the findings for psychological benefits are not as robust as compared to physical health, as there were inconsistent results that participants in expressive writing condition will have significant greater improvement than those in control condition [2], [13]. 
From a therapeutic perspective, writing intervention often serves as a non-verbal tool for supplementing traditional psychotherapy [14] that enables clients to restructure and make sense of their experience. Expressive writing is a low cost and time efficient psychological intervention that is applicable to both clinical and non-clinical populations [13]. It is a self-help technique that does not directly involve the presence of psychotherapists, and it allows internal dialogue with oneself. Furthermore, this writing technique provides opportunity for introspection and catharsis without fear of evaluation and judgment of others [15]. Expressive writing also helps individuals to explore their inner psychological resources, such as imagination and creativity to facilitate the integration of both the emotional and cognitive components of life experiences [16]. Hence, expressive writing intervention contains high therapeutic potential for individuals to heal from traumatic as well as stressful experience.

However, it was also suggested that writing about negative experience might be emotionally demanding for some of the individuals [17], because there would be an immediate increase in negative affect, distress and negative physical symptoms in expressive writing condition compared to control condition when individuals need to confront their unpleasant experience for the first time [1], even though the negative effects will diminish over time [18]. Another method of expressive writing which focuses on positive life experience was introduced with expectation that it might increase the positive feelings and eventually improve the sense of well-being [19]. One of the possible underlying mechanisms might be that positive experience writing helps to recover good memories that are suppressed, and this enables individuals to gain meaning and purpose from their disclosure writing [18]. The development is also related to the positive psychology movement, in which many psychological interventions are developed with the focus on wellbeing, personal strength and positive emotions [20]. It was discovered that participants who wrote about intensely positive experiences would have significantly more positive moods and fewer health center visits compared to participants in control condition [19], and it was also evident that writing out one's best possible future selves was less upsetting than writing about trauma and can lead to significantly better subjective wellbeing [21]. These findings suggested that disclosure writing might not necessarily need to be intensely trauma-focused to be effective for individuals, and the underlying mechanism by which expressive writing operates is also challenged [22].

Theoretical framework and literature review

The main difference between positive experience writing (PEW) and negative experience writing (NEW) is the affect, or feeling, that is induced during the cognitive process of engaging in expressive writing [19]. Individuals who wrote about intensely negative experiences might have high intensity negative feelings whereas individuals who wrote about positive experiences might have high intensity positive feelings after writing [23]. There are two possible pathways of this mechanism, in which one of the pathways is through the systematic-heuristic model of information processing [24]: the narration of negative experiences leads to an in-depth analysis of thoughts and feelings because negative emotions are often associated with deliberate systematic processing of information, positive emotions elicited from PEW often leads to heuristic approach, whereas an individual might use mental shortcuts to interpret their experiences, hence less likely to gain deep insight into their experience [19].

Nevertheless, it was argued that the systematic-heuristic model might not accurately capture the essence of PEW, as engaging in PEW involves significantly higher numbers of insightful words such as "understand, realize and reason" compared to NEW and control condition [25]. Therefore, another pathway model of affective and cognitive processing, "broaden and build" model of positive emotion, was introduced; this model suggested that positive examination of internal state might broaden an individual's coping and cognition processes, enabling one to avoid negative rumination and cognitive biases that often lead to onset of depression [11]. This is because individuals experiencing positive emotions would adopt a global mindset in the attentional process [26], and it led them to pay more attention to the whole picture of the experience, without fixating or ruminating on certain details [27]. Alternatively, individuals who experience negative emotions such as anger and distress tend to narrow their attentional focus on particular information, having greater difficulties in noticing and overcoming the emotional adversity [28].

This model also suggested the broadening of thought processes triggered by previous positive experience might facilitate subsequent coping processes, enhancing an individual's psychological well-being over time [29] by creating an upward spiral that serves as a buffer for depressed mood [30]. This is similar to the building aspect of the model, which suggested that the broadening process can lead to enduring and permanent changes in one's personal coping resources and cultivating resilience [31]; thereby, the repeated practice PEW might eventually reduce the depressive symptoms, enhance one's emotional well-being, increase positive affect and serve as a coping resource for individuals to cope with their life events [32].

While NEW was often reported to predict better psychological and physical outcomes than control condition [1], [2] there is still insufficient evidence that supported the efficacy of PEW on the same features. 
At the same time, studies comparing the effectiveness of PEW and NEW also showed contradictory findings [19], [25]. For instance, a significant increase in positive emotions in PEW condition compared to NEW and control condition among participants who experienced relationship dissolution [33]; while another study reported that PEW was inferior to NEW in reducing anxiety and stress for participants with mild mood disorders [22]. In addition, while many studies about PEW and NEW were conducted in laboratory settings, some studies have suggested that online writing intervention might be more convenient and accessible to a larger population than laboratory intervention [22], [34], especially during the current COVID-19 pandemic [35].

This current study attempts to fill in the previous gap by introducing online writing intervention with two types of expressive writing and one control condition to undergraduates in a private university in Malaysia. The participants were taken from this group because they were reported to be exposed to the highest risks of developing depressive symptoms among young adults in the country of Malaysia [36]. They are constantly distressed by issues such as academic performance, financial constraint, adjustment problems and lack of positive coping strategy. Hence, it is important to determine which type of disclosure writing intervention can serve as a therapeutic tool for enhancing their mental health, and possibly reduce the rate of getting clinical depression during their tertiary education. Moreover, this research might also provide some insights on the pathways by which PEW and NEW operates, hence contributing to the literature of theoretical mechanisms of expressive writing.

\section{RESEARCH METHOD}

\subsection{Design of the experiment}

Nonequivalent control group pre-test post-test design was used to investigate the effectiveness of expressive writing on reducing the depressive symptoms among students of a private university in Malaysia. The design was chosen due to its feature in comparing the depressive symptoms scores before and after receiving the treatment, and also compared the scores differences to the nonequivalent control group [37]. However, there was no random assignment in this study due to the constraint of the online research platform as participants were allowed to choose their own slots.

The independent variable (IV) in this study was the expressive writing, which consisted of three levels: positive experience writing (PEW), negative experience writing (NEW) and control writing (CW) conditions. The IV manipulation was conducted between-subjects as participants will be assigned with only one of the conditions, and required to perform the same writing condition on the online worksheet for a total of three sessions. The primary dependent variable (DV) was the depressive symptoms, which was measured at the beginning of the first session of writing intervention (pre-test) and at the end of the third session (posttest). In addition, the participants' affect was included as a manipulation check for the DV. Affect was measured by employing the positive affect and negative affect schedule (PANAS) [38] prior to and after each writing session.

\subsection{Materials}

Smyth and Pennebaker [39] define expressive writing as the technique for individuals to write about their thoughts and feelings about traumatic experiences or personal issues freely, without focusing on grammatical or spelling errors of the writing. Participants were required to perform the expressive writing intervention for around 15-20 minutes on the online worksheet generated from the instructions of previous research [19], [18], [40] for three sessions on three consecutive weeks. The same instructions were applied for all three sessions of this writing intervention.

The writing instructions for each condition that were adopted from past research shown are:

- Positive experience writing (PEW): "For the next three sessions, I would like you to think of the most wonderful experience or experiences in your life, happiest moments, ecstatic moments, moments of rapture, perhaps from being in love, or from listening to music, or suddenly "being hit' by a book or painting or from some great creative moment. Choose one such experience or moment. Try to imagine yourself at that moment, including all the feelings and emotions associated with the experience. Now write about the experience in as much detail as possible trying to include the feelings, thoughts, and emotions that were present at the time. Please try your best to re-experience the emotions involved" [19].

- Negative experience writing (NEW): "For the next three sessions, I would like for you to write about your very deepest thoughts and feelings about the most traumatic experience of your entire life. In your writing, I'd like you to really let go and explore your very deepest emotions and thoughts. You might link your topic to your relationships with others, including parents, lovers, friends, or relatives. You may also want to link your experience to your past, your present, or your future, or to who you have been, who you 
would like to be, or who you are now. You may write about the same general issues or experiences in all days of writing, or on different experiences each day. Don't worry about spelling, sentence structure, or grammar. The only rule is that once you begin writing, continue to do so until your time is up" [18].

- Control writing $(\mathrm{CW})$ : "For the next three sessions, I would like you to write today about what you did yesterday from the time you got up until the time you went to bed. In your writing, I want you to be as objective as possible about how you use your time. I am not interested in your emotions or opinions. Rather, I want you to be as objective as possible" [40].

The beck depression inventory (BDI) was used to measure the cognitive and affective component of depressive symptoms in both clinical and non-clinical populations [41]. This scale was administered to the participants at pre- and post-treatment, in order to examine the change in depressive symptoms between groups. The scale consists of 21-items inventory to measure an individual's current level of depressive symptoms. Individuals had to choose one of the four statements that best describe themselves with scores ranging from 0 to 3 , depending on the severity of the symptoms. The scale has high internal consistency (Cronbach's alpha=.87) and high two-week test-retest reliability $(r=.90)$ for non-psychiatric university students [42].

Positive and negative affect schedule [38]. Russell [43] defined affect as the non-conscious process of experiencing feeling or emotion, which affects the perception, cognition and behavior. The PANAS was used to measure the mood changes before and after the writing, in order to check the manipulation whether the type of expressive writing would induce the intended positive and negative emotions [25]. There were a total of 20-items in this scale, and participants were asked to indicate how they are feeling at this moment on a 5-point Likert scale, ranging from 1 (Very slightly or not at all) to 5 (Very much). This scale has high internal consistency of .84 to .90 , while the reliability for PA and NA are .89 and .85 respectively in nonclinical samples [44].

Demographic questionnaire. Information of participants including age, gender, nationality, and ethnicity, current participation in counseling or psychotherapy sessions and self-indicate English comprehension level was collected at the baseline.

\subsection{Participants}

A total of 88 undergraduate psychology students (11 males, 77 females) ranging from age 18 to 24 years old $(M=21.31, S D=1.41)$ were recruited from a private university in Malaysia. Participants were all recruited through an experimental portal called IPSY, which is the university's online experimental recruitment platform. Only 86 participants were included in the final sample because there were two participants who withdrew from the study due to absenteeism of one of the writing sessions. Purposive sampling was utilized in order to recruit them, where the opportunity to participate was advertised to undergraduates of the private university on the social media platform such as through the private university's intake groups in Facebook and WhatsApp. The participants were guided to log on to their portal account and sign up for the research experiment in the portal. A screening test would be provided to the participants prior to online experiment session, to check if participants did fulfill the criteria of (a) Should not be diagnosed with any severe psychological disorders; (b) Must not currently participate in any counseling or psychotherapy sessions; (c) Have been exposed to a traumatic or stressful experience more than one month ago. The study was approved by the university's ethical review board. Each participant received a $0.75 \%$ of course extra credits as compensation in their semester for completing three sessions of the online writing intervention.

\subsection{Procedure}

After completion of the screening test, participants were assigned with one of the conditions, which were PEW ( $n=31)$, NEW $(n=32)$ and CW $(n=23)$ conditions. At the beginning of the study, participants received a link to access Google Form for the first session of the study through their emails that were registered under their IPSY profiles. All participants were required to complete the study by using their own electronic gadget within 24 hours. At the first page of the form, participants were given an informed consent about the purpose and nature of the study. They were able to indicate their voluntary participation at the end of the first page. Next, they completed the demographic questionnaire, followed by the pretreatment measure of BDI and prewriting measure of PANAS. After completing the questionnaires, they then performed the writing intervention by typing the content in the space provided below the respective instructions of their conditions. There were no restrictions on the word limit for the length of their writing. In the next page, they filled in a post-writing assessment of PANAS. Lastly, they may click on the "Submit" button to record their responses for the first session.

The links for the second session were sent to participants via email one week after they completed the first session. In the second session, participants were required to perform the same writing condition with 
the measurement of pre- and post- writing PANAS. One week after the second session, the links to access the third writing session were sent to participants via email. In the third session, same writing instructions were applied to the participants of respective conditions while there were also pre- and post- writing assessments of PANAS. Upon completing the last session of writing, the post-treatment BDI questionnaire was filled in by participants. All participants were thanked for their participation at the end of the Google Form.

\section{RESULTS AND DISCUSSION}

\subsection{Baseline data}

Baseline comparisons among three writing groups were taken on the measure of BDI and PANAS. A series of one-way ANOVAs showed that there were no significant differences in depressive symptoms and negative affect between PEW, NEW and CW groups (ps>.05) at baseline. However, there was a significant difference in positive affect between PEW, NEW and CW groups, $F(2,83)=5.42$, p=.006. Post hoc analysis using Tukey HSD showed that the positive affect in Group PEW is significantly lower than Group NEW $\left(\mathrm{M}_{\text {diff }}=-5.40, S E=1.84, \mathrm{p}=.012\right)$ and Group $\mathrm{CW}\left(\mathrm{M}_{\text {diff }}=-5.43, S E=2.01, \mathrm{p}=.023\right)$ at baseline.

Table 1 indicates the multiple comparisons of positive affect between Group PEW, NEW and CW at baseline. Thus, a two-way ANOVA would be conducted to check whether the difference in baseline scores would affect the manipulation of the intended positive affect in writing groups, while Table 2 shows the complete mean scores of the variables in the baseline.

Table 1. Turkey HSD comparisons for positive affect at baseline

\begin{tabular}{lccc}
\hline Comparisons & Mdiff & SE & $\mathrm{p}$ \\
\hline PEW vs. NEW & -5.40 & 1.84 & .012 \\
PEW vs. CW & -5.43 & 2.01 & .023 \\
NEW vs. CW & -0.33 & 2.00 & 1.000 \\
\hline
\end{tabular}

Table 2. Baseline mean scores of BDI, PANAS (positive) and PANAS (negative) by experimental group

\begin{tabular}{ccccccc}
\hline & \multicolumn{2}{c}{ Group PEW } & \multicolumn{2}{c}{ Group NEW } & \multicolumn{2}{c}{ Group CW } \\
& M & SD & M & SD & M & SD \\
\hline BDI & 12.36 & 7.00 & 12.94 & 17.00 & 15.13 & 11.27 \\
PANAS (P) & 22.35 & 7.18 & 27.75 & 7.74 & 27.78 & 6.88 \\
PANAS (N) & 21.35 & 10.21 & 23.94 & 9.63 & 25.74 & 9.68 \\
\hline
\end{tabular}

\subsection{Manipulation check for positive affect}

This study expected that different expressive writing groups would induce different intensity of positive and negative affect after the writing intervention. This manipulation was crucial in examining whether the induced affect would contribute to the effectiveness of expressive writing in reducing depressive symptoms. Based on the manipulation, this study expected that the increase in positive affect rating would be the highest among Group PEW, compared to Group NEW and Group CW. A 3X3X2 (Condition X Session X Time) ANOVA was conducted to examine the changes in positive affect at prewriting and post-writing of each of the sessions. Assumption of normality was not met (ps<.05), while assumption of sphericity and assumption of homogeneity of variances was assumed (ps>.05). Table 3 shows the mean scores of PANAS (Positive) for three sessions.

Table 3. Mean scores for PANAS (P) ratings before and after the writing intervention by sessions

\begin{tabular}{lcccccc}
\hline & \multicolumn{2}{c}{ Session 1 } & \multicolumn{2}{c}{ Session 2 } & \multicolumn{2}{c}{ Session 3 } \\
& M & SD & M & SD & M & SD \\
\hline Group PEW & & & & & & \\
Before & 22.35 & 7.18 & 22.32 & 7.80 & 23.03 & 8.38 \\
After & 29.39 & 7.71 & 26.97 & 9.18 & 27.39 & 8.82 \\
Group NEW & & & & & & \\
Before & 27.75 & 7.74 & 26.75 & 9.58 & 23.88 & 8.39 \\
After & 23.94 & 7.56 & 22.66 & 9.39 & 22.72 & 9.37 \\
Group CW & & & & & & \\
Before & 27.78 & 6.88 & 25.22 & 8.06 & 26.61 & 7.38 \\
After & 28.52 & 7.75 & 24.57 & 8.72 & 25.96 & 9.10 \\
\hline
\end{tabular}

A significant interaction between time and expressive writing on positive affect was detected, $F(2,83)=30.09, \mathrm{p}<.001, \eta_{p}^{2}=.420$, indicating there was a significant change in positive affect from prewriting to postwriting as a function of different writing group. Simple main effects analyses using Bonferroni 
correction revealed that the increased in positive affect was significant in Group PEW $\left(M_{\text {diff }}=5.34, S E=0.78\right.$, $\mathrm{p}<.001)$ and decreased in positive affect was significant in Group NEW $\left(M_{\text {diff }}=-3.02, S E=0.77, p<.001\right)$, but no significant difference in positive affect between prewriting and postwriting in Group CW $\left(M_{\text {diff }}=0.19\right.$, $S E=0.90, \mathrm{p}=.835)$. Furthermore, after the writing intervention, the positive affect in Group PEW was significantly higher than Group NEW, $M_{\text {diff }}=4.81, S E=1.91, \mathrm{p}=.042$, but not significantly higher than Group $\mathrm{CW}, M_{\text {diff }}=1.57, S E=2.09, \mathrm{p}=1.000$, hence the hypothesis 1 that stated that positive affect will be the highest in Group PEW at postwriting was not supported. In addition, there was no significant difference in the positive affect between Group CW and Group NEW, $M_{\text {diff }}=3.24, S E=2.08, \mathrm{p}=.365$. Refer to Table 4 for Bonferroni comparisons of positive affect at post-writing between Group PEW, NEW and CW.

Table 4. Bonferroni comparisons for positive affect at post-writing

\begin{tabular}{cccc}
\hline Comparisons & $\mathrm{M}_{\text {diff }}$ & $\mathrm{SE}$ & $\mathrm{p}$ \\
\hline PEW vs. NEW & 4.81 & 1.91 & .042 \\
PEW vs. CW & 1.57 & 2.09 & 1.000 \\
CW vs. NEW & 3.24 & 2.08 & .365 \\
\hline
\end{tabular}

\subsection{Manipulation check for negative affect}

For negative affect manipulation, this study postulated that negative affect will be the highest in Group NEW, as compared to Group PEW and CW after participants performed the writing. A three-way ANOVA (Condition X Session X Time) was conducted to analyze the change of negative affect at prewriting and postwriting of each session. The assumption of normality was not met (ps<.05) Refer to Table 5 for the mean scores of PANAS (Negative) for three sessions.

Table 5. Mean scores for PANAS (N) ratings before and after the writing intervention by sessions

\begin{tabular}{ccccccc}
\hline & \multicolumn{2}{c}{ Session 1 } & \multicolumn{2}{c}{ Session 2 } & \multicolumn{3}{c}{ n 3 } & \\
& $\mathrm{M}$ & $\mathrm{SD}$ & $\mathrm{M}$ & $\mathrm{SD}$ & $\mathrm{M}$ & $\mathrm{SD}$ \\
\hline Group PEW & & & & & & \\
Before & 21.35 & 10.21 & 19.13 & 7.62 & 16.19 & 6.87 \\
$\quad$ After & 15.81 & 7.54 & 14.48 & 5.28 & 13.68 & 5.88 \\
Group NEW & & & & & & \\
$\quad$ Before & 23.94 & 9.63 & 20.16 & 6.88 & 21.22 & 8.94 \\
$\quad$ After & 25.75 & 9.38 & 24.81 & 9.64 & 23.31 & 8.92 \\
Group CW & & & & & & \\
Before & 25.74 & 9.68 & 24.70 & 9.70 & 22.09 & 8.59 \\
After & 19.57 & 8.99 & 21.04 & 11.30 & 17.83 & 8.52 \\
\hline
\end{tabular}

The results showed that there was a significant interaction between time and expressive writing on negative affect, $F(2.83)=25.77, \mathrm{p}<.001, \eta_{p}^{2}=.383$. A simple main effect analyses revealed that negative affect was increased significantly in Group NEW after the writing intervention, $M_{\text {diff }}=2.85, S E=0.81, \mathrm{p}=.001$; while there was significant reduction in negative affect in both Group PEW $\left(M_{\text {diff }}=-4.24, S E=0.82, p<.001\right)$ and Group CW $\left(M_{d i f f}=-4.70, S E=0.95, \mathrm{p}<.001\right.$. At post-writing, the negative affect in Group NEW was significantly higher than in Group PEW $\left(M_{\text {diff }}=9.97, S E=1.79, \mathrm{p}<.001\right)$ and Group CW $\left(M_{\text {diff }}=5.15, S E=1.94\right.$, $\mathrm{p}=.029$ ). Thus, hypothesis 2 which proposed that the negative affect will be the highest in Group NEW, compared to Group PEW and Group CW after the writing intervention was supported. Intriguingly, Group $\mathrm{CW}$ had significantly higher negative affect than Group PEW at the post-writing, $M_{d i f f}=4.82, S E=1.96$, $\mathrm{p}=.047$. Table 6 shows the Bonferroni comparisons of negative affect at post-writing between Group PEW, NEW and CW.

Table 6. Bonferroni comparisons for negative affect at post-writing

\begin{tabular}{cccc}
\hline Comparisons & $\mathrm{M}_{\text {diff }}$ & $\mathrm{SE}$ & $\mathrm{p}$ \\
\hline NEW vs. PEW & 9.97 & 1.79 & .000 \\
NEW vs. CW & 5.15 & 1.94 & .029 \\
CW vs. PEW & 4.82 & 1.96 & .047 \\
\hline
\end{tabular}

Int. J. Public Health Sci., Vol. 10, No. 2, June 2021: 433 - 444 


\subsection{Depressive symptoms}

Table 7 suggests that at the Time 1 (pre-treatment), participants in the Group CW had the highest score in depressive symptoms $(M=15.13, S D=11.27)$, followed by participants in Group NEW $(M=12.94$, $S D=7.00)$, and in Group PEW $(M=12.36, S E=7.00)$; At Time 2 (post-treatment), participants in Group PEW had the lowest depressive symptoms $(M=8.52, S E=7.65)$, followed by participants in Group NEW $(M=12.22$, $S E=6.28)$ and in Group CW $(M=14.74, S E=10.73)$.

Table 7. Mean scores for BDI at pretreatment and post-treatment across writing groups

\begin{tabular}{ccccc}
\hline & \multicolumn{2}{c}{ Pre-treatment } & \multicolumn{2}{c}{ Post-treatment } \\
& $\mathrm{M}$ & $\mathrm{SD}$ & $\mathrm{M}$ & $\mathrm{SD}$ \\
\hline Group PEW & 12.36 & 7.00 & 8.52 & 7.65 \\
Group NEW & 12.94 & 7.00 & 12.22 & 6.28 \\
Group CW & 15.13 & 11.27 & 14.74 & 10.73 \\
\hline
\end{tabular}

Shapiro-Wilk analysis showed that the assumptions of normality were assumed for all levels of the between-subjects variable (ps>.05), except for the post-treatment BDI scores in PEW condition, W(31) $=0.91$, $\mathrm{p}=.010$. Levene's test showed that the assumption of homogeneity of variance was not met for pretreatment BDI scores, $F(2,83)=4.96, p=.009$ and post-treatment BDI scores, $F(2,83)=4.34, p=.016$. Hence, the results should be interpreted with caution.

The results of $3 \mathrm{X} 2$ (Condition $\mathrm{X}$ Time) mixed ANOVA showed that there was a significant difference of depressive symptoms between pretreatment and post-treatment, $F(1,83)=8.54, p=.004, \eta_{p}^{2}=$ .093, while the effect size was moderate [45]. The depressive symptoms scores in post-treatment were significantly lower than the scores in pretreatment, $\mathrm{M}_{\text {diff }}=-1.65, \mathrm{SE}=0.57, \mathrm{p}=.004$. However, there was no significant main effect of type of expressive writing on depressive symptoms, $F(2,83)=2.188, p=.119$, $\eta_{p}^{2}=.050$.Thus, there were no significant differences in average depressive symptoms scores between pretreatment and post-treatment of Group PEW, NEW and CW.

There was a significant interaction effect of time and expressive writing on depressive symptoms, $\mathrm{F}(2,83)=3.95, \mathrm{p}=.023, \eta_{\mathrm{p}}^{2}=.087$, indicating there was a significant change in depressive symptoms over time as a function of type of expressive writing. The effect size of this interaction effect was moderate. Simple main effects analyses using Bonferroni correction showed that Group PEW showed significant reduction in depressive symptoms from pretreatment to post-treatment, $\mathrm{M}_{\text {diff }}=-3.84, \mathrm{SE}=0.93, \mathrm{p}<.001$. However, such reduction was not observed in either Group NEW $\left(\mathrm{M}_{\text {diff }}=-0.72, \mathrm{SE}=0.92, \mathrm{p}=.435\right)$ or Group CW $\left(\mathrm{M}_{\text {diff }}=-\right.$ $0.39, \mathrm{SE}=1.08, \mathrm{p}=.718$ ).

Furthermore, at post-treatment, participants in Group PEW scored significantly lower than Group $\mathrm{CW}\left(\mathrm{M}_{\mathrm{diff}}=-6.22, \mathrm{SE}=2.25, \mathrm{p}=.021\right)$. Hence, hypothesis 3 that stated participants who engage in Group PEW would have significantly greater reduction in depressive symptoms than participants who engage in Group $\mathrm{CW}$ was supported. However, there was no significant difference in depressive symptoms between Group NEW and Group $\mathrm{CW}\left(\mathrm{M}_{\mathrm{diff}}=2.58, \mathrm{SE}=2.23, \mathrm{p}=.751\right)$ at post-treatment, indicating hypothesis 4 that participants in Group NEW would have significantly greater reduction in depressive symptoms than participants in Group CW was not supported. In addition, participants in Group PEW did not score significantly lower than Group NEW ( $\left.\mathrm{M}_{\mathrm{diff}}=-3.64, \mathrm{SE}=2.06, \mathrm{p}=.241\right)$. Thus, hypothesis 5 that Group PEW would have significantly greater reduction in depressive symptoms compared to Group CW and Group NEW was also not supported. Refer to Table 8 for Bonferroni comparisons of depressive symptoms at posttreatment between Group PEW, NEW and CW.

Table 8. Bonferroni comparisons for depressive symptoms at post-treatment

\begin{tabular}{lllc}
\hline Comparisons & $\mathrm{M}_{\text {diff }}$ & SE & $\mathrm{p}$ \\
\hline PEW vs. CW & -6.22 & 2.25 & .021 \\
PEW vs. NEW & -3.64 & 2.06 & .241 \\
NEW vs. CW & -2.58 & 2.23 & .751 \\
\hline
\end{tabular}

\subsection{Discussion}

The results of the first hypothesis testing showed that there were no significant differences in positive affect between PEW and CW condition, which was inconsistent with past studies [23], [25]. This finding suggested that $\mathrm{CW}$ condition might provide positive emotional benefits similar to PEW because writing about daily routines and time management will allow individuals to have greater sense of efficacy in scheduling and managing their life [22], which was shown in the writing instruction of "I want you to be as 
objective as possible about how you use your time." Therefore, individuals engaged in control writing tasks might have higher levels of self-efficacy, which was subsequently associated with an elevated level of positive mood state [46]. In addition, participants in PEW will have significantly higher positive affect than those in NEW, suggesting that positive affect was elicited through re-visiting significant positive events in the past [19]. In the process of writing about past positive events, they might re-experience feelings of joy, enjoyment or sense of accomplishment and that will lead to higher intensity of positive affect compared to those who wrote about past traumatic events.

For the second hypothesis of negative affect manipulation, the findings indicated that NEW induced significantly higher negative affect than PEW and CW conditions at post writing, which was consistent with the theoretical assumption for, broaden and build models. As suggested by past research [1], individuals engaged in NEW will have high initial emotional engagement when they need to confront their previous negative past experience by writing about "deepest thoughts and feelings about the most traumatic experience of your entire life" for the first time. As compared to writing about past positive experiences or daily routine, the writing of traumatic experiences was usually more personally stressful and sensitive; hence often elicit high emotional arousal [47]. For instance, individuals would often experience feelings of nervousness, sadness or even frustration in the writing sessions that contributed to the high intensity of negative affect [8]. Pascual-Leano and colleagues (2016) also mentioned that this negative arousal was part of the process of meaning-making, as there will be an increase in emotional arousal when individuals discover new perspectives or meaning towards their past experiences in their writing.

Furthermore, the findings showed that participants in PEW condition had significantly greater reduction in depressive symptoms compared to those in $\mathrm{CW}$ condition, which was consistent with the third hypothesis based on broaden and build models. PEW was the only condition to have a significant reduction of depressive symptoms from pretreatment to post-treatment. This is because the positive affect that was induced during the writing about past positive experience will broaden the cognitive processes of the individuals, enabling ones to have more flexible thinking in solving problems and building greater psychological resilience in the face of adversity [48]. Hence, the increase in coping resources and building of resilience will buffer the depressive symptoms among individuals who are vulnerable to stress [49]. As an illustration, individuals were able to gain positive insights into their past experience and take a broader perspective on their emotional problems. They tend to integrate their diverse experience as part of their life rather than fixate or ruminate on certain negative details [27], thus more likely to have reduction in depressive symptoms. In addition, the PEW intervention might also provide the psychological benefits through creating positive upward spirals for emotional well-being [32] as the effects of the positive emotions will accumulate through continuous practice of the writing for three sessions in this study.

Although PEW condition did not induce significantly higher positive affect than CW condition after writing, the results of post-treatment showed that PEW was still superior to $\mathrm{CW}$ in reducing depressive symptoms. This suggested that even though positive affect is necessary for broadening of thought and building of personal resources, the affect might not be the only contributing factor for individuals to experience cognitive broadening and gain psychological benefits from the process. Study [50] mentioned that one's use of global or local focus might be a mediating factor in the broaden and build model. For instance, individuals who engage in CW condition might be less likely to adopt a global focus as compared to PEW condition because the control writing instructions were emphasized on reiterating daily routine as objective and detailed as possible. It was unlikely that individuals would explore their daily experience from a wider perspective or discover new meanings through objectively writing about their daily routine [4]. Therefore, the cognitive broadening might not occur without the presence of global focus in the writing intervention, which subsequently would not lead to the building or accumulation of personal coping resources that is crucial in reducing depressive symptoms.

In addition, this study also found out that the fourth hypothesis was not supported since NEW did not report a significant greater reduction in depressive symptoms as compared to $\mathrm{CW}$ condition. The findings contradicted the previous literature [11], [13], [47] that highlighted the efficacy of NEW or trauma-focused expressive writing on reducing psychological symptoms in both clinical and non-clinical populations as compared to control writing condition. One explanation might be the negative affect that was induced during NEW condition would narrow individuals' thought-action repertoire and create a local focus approach in making sense of their experience during the structured writing [28]. When the thought-action repertoire is narrowed, individuals will have fewer options and resources to cope with their stressful life events, eventually the level of depressive symptoms will not be reduced. Notably, Moberly and Watkins [51] mentioned the negative affect is often associated with the engagement in ruminative self-focus. Thus, the tendency to ruminate will perpetuate negative thinking patterns and reduce one's ability to cope with their adverse past experience [52], hence the symptoms reduction might not be effective through NEW. 
The last hypothesis was not supported as participants in PEW condition did not report significantly lower depressive symptoms as compared to NEW condition at post-treatment. This finding is not consistent with the theoretical model that extrapolated the PEW would lead to greater reduction in depressive symptoms as compared to NEW [31]. One of the explanations might be while the negative affect that was induced during NEW will lead to a self-focus and self-ruminating tendency; the repeated exposure of NEW to individuals might actually produce a habituation for these negative emotional responses as stated in the exposure hypothesis [53]. As an illustration, individuals would have less intense negative arousal when they wrote about their past stressful or traumatic experience for the second or third time [18] and the tendency to ruminate might be reduced as well. Hence, this might explain the depressive symptoms of NEW did not differ significantly with PEW at post-treatment, since the negative responses of NEW might diminish through repeated practice.

Another explanation might be the induced negative affect during NEW will actually lead to an indepth analysis of personal experience; hence individuals would be able to gain more insights into their past experience and integrate their negative experience [19], [24]. This explanation highlighted that perhaps the positive and negative affect plays a different role for individuals to process their experience during the writing sessions. For instance, positive affect elicited during PEW might lead to cognitive broadening and building of coping resources while the negative affect elicited from NEW would lead to more deliberate effort of analyzing as well as integrating past negative experience. Hence, this might explain why PEW did not have significantly lower depressive symptoms than NEW at post-treatment, as NEW might still function as an intervention in preserving individual's mental well-being, even though it did not significantly reduce one's symptoms throughout the sessions.

One of the limitations of this study is the small sample size of participants. The study had recruited 86 undergraduates through the experiment portal of the private university in Malaysia, but it still did not reach the initial requirement of 120 participants to meet the power of 0.80 and the effect size of $f=0.25$ [54] in order to sufficiently detect the effect of expressive writing on depressive symptoms. The time constraint in the data collection was the major reason for insufficient sample size in this study, as the researcher had only two weeks to recruit participants before the online experiment started. Further study can recruit participants from other universities to increase the sample size as well as the diversity of the participants. This is because all of the participants in this study were recruited from the same major of the private university, and the lack of diversity in the subject pools might lead to unrepresentativeness of the population at large [55]. Hence, by recruiting participants from various majors in different universities, the results can be more generalizable to the general population of undergraduates.

Another clear limitation in this study is the lack of randomization to prevent selection bias in the assignment of experimental conditions, which is a common issue faced in online study [34]. The major reason that caused the lack of randomization is the technical setup of the experimental portal for this research. In this study, all participants were recruited from the same experimental portal that was used in the university. They were allowed to sign up for the slots that they wanted in the study. Hence, the allocation of treatment conditions was based on the session that they signed up. For instance, if 1A slot is allocated for PEW condition, participants who were signed up in that slot will undergo writing about personal positive experience. Therefore, future research can utilize a computerized method that was described in a study [56], in which there will be a list of randomly generated allocation of treatment conditions that is stored in the computer prior to the data collection phase. Once participants are signed up for the experiment and passed the screening test, researchers will then input the participants' codes into the list and assign respective treatment conditions to the participants.

Lastly, a potential area for future research is to examine the mediating role of global and local focus in the process of engaging in structured writing. As shown in the findings, the induced positive or negative affect did not sufficiently explain the efficacy of both PEW and NEW as compared to CW in the writing sessions. Therefore, it is crucial to investigate the underlying psychological mechanism of decreased psychological symptoms of PEW because this will assist therapists or interventionists to determine whether this intervention is suitable for the use of public as well as for individuals with psychological conditions.

This research has compared the effectiveness of writing about personal positive experience, personal negative experience as well as daily routine (control topic) in reducing depressive symptoms among undergraduates. While past studies have mainly emphasized on the expressive writing of traumatic experiences, this study introduced Burton and King's [19] instruction of positive experience writing into the online experimental setting. By using broaden and build models as the theoretical framework, this study found out that positive affect might play an important role in reducing individuals' depressive symptoms by broadening cognition, building coping resources and cultivating resilience. However, there might be other potential mediating factors involved in this model such as the use of local or global focus in one's attentional process that need to be addressed in future research. This study has also extended the role of broaden and 
build models from promoting positive emotional health into a more clinical-based framework that can help to reduce symptoms of depression for those who are vulnerable to stress through emotional disclosure writing.

Furthermore, the findings of this research have provided some preliminary insights into the potential value of the online practice of writing about personal positive experience in reducing depressive symptoms among undergraduates. The theoretical mechanism of writing about positive emotional experience is similar to the positive psychology technique such as the process of savoring and reminiscence [57], while the writing process is more structured and insightful for individuals to practice [18]. Hence, the educational sector can introduce the positive experience writing practice into university settings, guiding individuals to write about their own positive experience in the online platform such as personal social media accounts to help alleviate the negative symptoms that might arise from one's stressful lifestyle. This writing technique might also serve as an effective coping strategy for individuals who are vulnerable to stress and anxiety, such as people who experienced loss of employment during COVID-19 pandemic. Furthermore, even though trauma-focused writing has been used primarily in narrative and exposure therapy [15], [14], therapists or interventionists could introduce an alternative of positive experience writing as a more strength-focused technique for clients who are uncomfortable to explore their traumatic or stressful past experience through traditional expressive writing.

\section{CONCLUSION}

All in all, this research was conducted to examine the effect of type of expressive writing on depressive symptoms among undergraduates. The findings showed that only participants who wrote about their personal positive experience have significant reduction in depressive symptoms from pretreatment to post-treatment, and have significant lower depressive symptoms than control writing condition. This may be due to the positive affect that was induced during the writing sessions broadened one's cognitive process and enabled one to have more coping resources to overcome emotional adversity. However, the positive affect might not be exclusively explaining the mechanism of broaden and build model, as the writing of positive experience did not elicit significantly higher positive affect than control writing condition. Furthermore, such reduction in depressive symptoms was not observed among participants who wrote about their personal negative experience, and this might be due to the induced negative affect that lead to fixation and rumination of the problem. However, it is important to note that these effects of negative affect might diminish over time, as the findings showed that the depressive symptoms of PEW and NEW did not differ significantly at post-treatment. Future research can be conducted to examine the mediating role of the use of global or local focus in the writing session, as well as examining the long-term psychological effect of type of expressive writing on mental health.

\section{ACKNOWLEDGEMENTS}

This study is funded by Internal Research Grant Scheme \#21-01-001, HELP University

\section{REFERENCES}

[1] K. A. Baikie and K. Wilhelm, "Emotional and physical health benefits of expressive writing," Advances in Psychiatric Treatment, vol. 11, no. 5, pp. 338-346, 2005.

[2] J. W. Pennebaker and C. K. Chung, "Expressive writing: Connections to physical and mental health," Oxford Handbook of Health Psychology, pp. 417-437, 2011.

[3] J. W. Pennebaker and S. K. Beall, "Confronting a traumatic event: Toward an understanding of inhibition and disease," Journal of Abnormal Psychology, vol. 95, no. 3, p. 274, 1986.

[4] M. E. Francis and J. W. Pennebaker, "Putting stress into words: The impact of writing on physiological, absentee, and self-reported emotional well-being measures," American Journal of Health Promotion, vol. 6, no. 4, pp. 280287, 1992, https://doi.org/10.4278/0890-1171-6.4.280.

[5] H. E. Smith., C. J. Jones, and M. Hankins., "The effects of expressive writing on lung function, quality of life, medication use, and symptoms in adults with asthma: A randomized controlled trial," Psychosomatic Medicine, vol. 77, no. 4, pp. 429-437, 2015.

[6] K. M. B. McGuire, M. A. Greenberg, and R. Gevirtz, "Autonomic effects of expressive writing in individuals with elevated blood pressure,” Journal of Health Psychology, vol. 10, no. 2, p. 197-209, 2005.

[7] R. J. Booth, K. J. Petrie, and J. W. Pennebaker, "Changes in circulating lymphocyte numbers following emotional disclosure: Evidence of buffering," Stress Medicine, vol. 13, no. 1, pp. 23-29, 1997.

[8] J. W. Pennebaker and M. E. Francis, "Cognitive, emotional, and language processes in disclosure," Cognition \& Emotion, vol. 10, no. 6, pp. 601-626, 1996.

[9] K. Klein and A. Boals., "Expressive writing can increase working memory capacity," Journal of Experimental Psychology: General, vol. 130, no. 3, p. 520, 2001. 
[10] J. M. Smyth, J. R. Hockemeyer, and H. Tulloch, "Expressive writing and post-traumatic stress disorder: Effects on trauma symptoms, mood states, and cortisol reactivity," British Journal of Health Psychology, vol. 13, no. 1, pp. 85-93, 2008.

[11] E. M. Gortner, S. S. Rude, and J. W. Pennebaker, "Benefits of expressive writing in lowering rumination and depressive symptoms," Behavior Therapy, vol. 37, no. 3, pp. 292-303, 2006.

[12] L. J. Barclay and D. P. Skarlicki, "Healing the wounds of organizational injustice: Examining the benefits of expressive writing," Journal of Applied Psychology, vol. 94, no. 2, p. 511, 2009.

[13] M. Reinhold, P. C. Bürkner, and H. Holling, "Effects of expressive writing on depressive symptoms-A metaanalysis," Clinical Psychology: Science and Practice, vol. 25, no. 1, p. e12224, 2018.

[14] L. L'Abate, “The use of writing in psychotherapy," American Journal of Psychotherapy, vol. 45, no. 1, pp. 87-98, 1991.

[15] G. Bolton, S. Howlett, C. Lago, and J. K. Wright, "Writing Cures: An Introductory Handbook of Writing in Counseling and Therapy," Hove: Brunner-Routledge, 2004.

[16] A. Lange, "Using writing assignments with families managing legacies of extreme traumas," Journal of Family Therapy, vol. 18, no. 4, pp. 375-388, 1996.

[17] J. M. Smyth and N. True e J. Souto, "Effects of writing about traumatic experiences: The necessity for narrative structuring," Journal of Social and Clinical Psychology, vol. 20, no. 2, pp. 161-172, 2001.

[18] J. W. Pennebaker, "Writing about emotional experiences as a therapeutic process," Psychological Science, vol. 8, no. 3, pp. 162-166, 1997.

[19] C. M. Burton and L. A. King, "The health benefits of writing about intensely positive experiences," Journal of Research in Personality, vol. 38, no. 2, pp. 150-163, 2004.

[20] M. E. Seligman, "Authentic happiness: Using the new positive psychology to realize your potential for lasting fulfillment," Simon and Schuster, 2004.

[21] L. A. King, "The health benefits of writing about life goals," Personality and Social Psychology Bulletin, vol. 27, no. 7, pp. 798-807, 2001.

[22] K. A. Baikie, L. Geerligs, and K. Wilhelm, "Expressive writing and positive writing for participants with mood disorders: An online randomized controlled trial," Journal of Affective Disorders, vol. 136, no. 3, pp. 310-319, 2012.

[23] H. Marlo and M. K. Wagner, "Expression of negative and positive events through writing: Implications for psychotherapy and health," Psychology and Health, vol. 14, no. 2, pp. 193-215, 1999.

[24] G. Bohner, S. Chaiken, and P. Hunyadi, "The role of mood and message ambiguity in the interplay of heuristic and systematic processing," European Journal of Social Psychology, vol. 24, no. 1, pp. 207-221, 1994.

[25] J. D. Kloss and S. A. Lisman, "An exposure-based examination of the effects of written emotional disclosure," British Journal of Health Psychology, vol. 7, no. 1, pp. 31-46, 2002.

[26] K. Gasper and G. L. Clore, "Attending to the big picture: Mood and global versus local processing of visual information," Psychological Science, vol. 13, no. 1, pp. 34-40, 2002.

[27] P. M. Niedenthal and S. Kitayama, "The heart's eye: Emotional influences in perception and attention," Academic Press, 2013. [Online]. Available: https://psycnet.apa.org/record/1994-97332-000.

[28] B. L. Fredrickson, "The role of positive emotions in positive psychology: The broaden-and-build theory of positive emotions," American Psychologist, vol. 56, no. 3, p. 218, 2001.

[29] F. G. Ashby and A. M. Isen, "A neuropsychological theory of positive affect and its influence on cognition," Psychological Review, vol. 106, no 3, p. 529, 1999.

[30] L. G. Aspinwall, "Rethinking the role of positive affect in self-regulation," Motivation and Emotion, vol. 22, no. 1, pp. 1-32, 1998.

[31] B. L. Fredrickson, "What good are positive emotions," Review of General Psychology, vol. 2, no. 3, pp. 300-319, 1998.

[32] B. L. Fredrickson and T. Joiner, "Positive emotions trigger upward spirals toward emotional well-being," Psychological Science, vol. 13, no. 2, pp. 172-175, 2002.

[33] G. W. Lewandowski Jr, "Promoting positive emotions following relationship dissolution through writing," The Journal of Positive Psychology, vol. 4, no. 1, pp. 21-31, 2009.

[34] M. Hirai, S. Dolma, L. L. Vernon, and G. A. Clum, "A longitudinal investigation of the efficacy of online expressive writing interventions for Hispanic students exposed to traumatic events: Competing theories of action," Psychology \& Health, vol. 35, no 12, pp. 1459-1476, 2020.

[35] T. R. Wind, M. Rijkeboer, G. Andersson, and H. Riper, "The COVID-19 pandemic: The 'black swan' for mental health care and a turning point for e-health," Internet Interventions, vol. 20, 2020.

[36] H. Elias, W. S. Ping, and M. C. Abdullah, "Stress and academic achievement among undergraduate students in Universiti Putra Malaysia," Procedia-Social and Behavioral Sciences, vol. 27, no. 2, pp. 646-655, 2011.

[37] D. A. Kenny, "A quasi-experimental approach to assessing treatment effects in the nonequivalent control group design," Psychological Bulletin, vol. 82, no. 3, pp. 345-362, 1975, https://psycnet.apa.org/doi/10.1037/00332909.82.3.345.

[38] D. Watson, L. A. Clark, and G. Carey, "Positive and negative affectivity and their relation to anxiety and depressive disorders," Journal of Abnormal Psychology, vol. 97, no. 3, p. 346, 1988.

[39] J. M. Smyth and J. W. Pennebaker, "Exploring the boundary conditions of expressive writing: In search of the right recipe," British Journal of Health Psychology, vol. 13, no. 1, pp. 1-7, 2008.

[40] J. W. Pennebaker, M. Colder, and L. K. Sharp, "Accelerating the coping process," Journal of Personality and Social Psychology, vol. 58, no. 3, p. 528, 1990. 
[41] A. T. Beck, A. J. Rush, and B. F. Shaw, "Cognitive therapy of depression,” New York: Guilford, 1979.

[42] S. L. Lightfoot and J. M. Oliver, "The beck inventory: Psychometric properties in university students," Journal of Personality Assessment, vol. 49, no. 4, pp. 434-436, 1985.

[43] J. A. Russell, "Core affect and the psychological construction of emotion," Psychological Review, vol. 110, no. 1, p. 145,2003

[44] J. R. Crawford and J. D. Henry, "The Positive and Negative Affect Schedule (PANAS): Construct validity, measurement properties and normative data in a large non-clinical sample," British Journal of Clinical Psychology, vol. 43, no. 3, pp. 245-265, 2004.

[45] D. Lakens, "Calculating and reporting effect sizes to facilitate cumulative science: A practical primer for t-tests and ANOVAs," Frontiers in Psychology, vol. 4, p. 863, 2013.

[46] A. Craig, N. Wijesuriya, and Y. Tran, "The influence of self-efficacy on mood states in people with spinal cord injury," ISRN rehabilitation, pp. 1-6, 2013

[47] S. J. Lepore, "Expressive writing moderates the relation between intrusive thoughts and depressive symptoms," Journal of Personality and Social Psychology, vol. 73, no. 5, p. 1030, 1997.

[48] S. Folkman and J. T. Moskowitz, "Positive affect and the other side of coping," American Psychologist, vol. 55, no. 6, p. 647, 2000.

[49] A. P. Wingo, G. Wrenn, and T. Pelletier, "Moderating effects of resilience on depression in individuals with a history of childhood abuse or trauma exposure," Journal of Affective Disorders, vol. 126, no. 3, pp. 411-414, 2010.

[50] C. M. Burton and L. A. King, "The health benefits of writing about positive experiences: The role of broadened cognition," Psychology and Health, vol. 24, no. 8, pp. 867-879, 2009.

[51] N. J. Moberly and E. R. Watkins, "Ruminative self-focus and negative affect: An experience sampling study," Journal of Abnormal Psychology, vol. 117, no. 2, p. 314, 2008.

[52] S. Nolen-Hoeksema and J. Morrow, "Effects of rumination and distraction on naturally occurring depressed mood," Cognition \& Emotion, vol. 7, no 6, pp. 561-570, 1993.

[53] Lepore, S. J and Smyth, J. M, "The writing cure: How expressive writing promotes health and emotional wellbeing," American Psychological Association, 2002. https://doi.org/10.1037/10451-000.

[54] F. Faul, E. Erdfelder, and A. Buchner, "Statistical power analyses using G*Power 3.1: Tests for correlation and regression analyses," Behavior Research Methods, vol. 41, no. 4, pp. 1149-1160, 2009.

[55] J. Henrich, S. J. Heine and A. Norenzayan, "Beyond WEIRD: Towards a broad-based behavioral science," Behavioral and Brain Sciences, vol. 33, no. 2-3, p. 111, 2010.

[56] G. N. Braucht and C. S. Reichardt, "A computerized approach to trickle-process, random assignment," Evaluation Review, vol. 17, no. 1, pp. 79-90, 1993.

[57] F. B. Bryant, C. M. Smart, and S. P. King., "Using the past to enhance the present: Boosting happiness through positive reminiscence," Journal of Happiness Studies, vol. 6, no. 3, pp. 227-260, 2005. 